\title{
Bericht der Arbeitsgruppe «Ligen und private Organisationen»
}

\author{
Rudolf Bruppacher und Jürg Barth \\ Gesundheitsligenkonferenz, Schanzeneggstrasse 1, 8039 Zürich
}

\begin{abstract}
Die Arbeitsgruppe «Ligen und private Organisationen» vereinigte 19 Teilnehmer, die ein weites Spektrum privater Organisationen, aber auch Universitätsinstitute und kantonale Verwaltungen repräsentierten. Im folgenden sind die aus der Sicht der Diskussionsleiter wichtigsten Ergebnisse zusammengefasst.
\end{abstract}

\section{Initiative eher vom einzelnen als von Organisationen} Die Prävention nimmt an Bedeutung zu; Initiativen in dieser Hinsicht sind notwendig, sind aber nach der Meinung der meisten Mitglieder der Arbeitsgruppe auch vorhanden. Private Organisationen sind dazu sicher prädisponiert. Sie sind weniger gebunden und in diesem Sinne aktionsfähiger als staatliche Organe. Aber auch von seiten der Mitglieder der Verwaltung kann die Initiative ergriffen werden. Man könnte vermutlich feststellen, dass die Initiative vom einzelnen ausgehen muss, in welcher Organisation er auch immer plaziert ist. Auch Einzelpersonen in Standesorganisationen können hier aktiver werden, zum Beispiel Parlamentsvertreter.

\section{Besondere Erwartungen an private Organisationen}

Die Diskussion drehte sich hauptsächlich um die Rolle der privaten Organisationen in der Prävention unter dem Stichwort «Erwartungen». Von privaten Organisationen wird erwartet, dass diese aktiver sein können, dass sie flexibler sind, freier sind, ohne Bürokratie arbeiten und damit auch gewisse Pionierarbeit leisten können. Sie gelten auch als gute Kontaktstellen zu besonderen Zielgruppen. Andererseits wurde festgehalten, dass Erwartungen der Öffentlichkeit die Struktur der Organisationen und ihre Funktionsweise nicht immer auch bestimmen.

\section{Probleme der Zusammenarbeit \\ Staat - private Organisationen}

Das zweite Stichwort, das die Arbeitsgruppe beschäftigte, war «Zusammenarbeit», Zusammenarbeit zunächst zwischen privaten Organisationen und dem Staat. Hier geht es um die Frage der Finanzierung und der Kontrolle. Es geht darum, gegenseitige Ängste abzubauen, Ängste, die andererseits sicher auch zwischen den privaten Organisationen existieren.

Vor allem die traditionellen Ligen und Gesundheitsorganisationen haben ein immaterielles Kapital, das auch im modernen Gesundheitswesen breiter genützt werden sollte. Auf der anderen Seite gibt es auch beim Staat brachliegende Strukturen, beispielsweise die
Gesundheitskommissionen, die reaktiviert werden und zur Prävention beitragen sollten.

\section{Zusammenarbeit zwischen Fachleuten}

Interdisziplinäre Expertengruppen sind sehr wichtig. Die Ärzte innerhalb der Arbeitsgruppe haben aber allerdings auch der Meinung Ausdruck gegeben, dass die Ärzteschaft im modernen Trend der breiten Prävention häufig übergangen oder beiseite gestellt wird. Dies ist, darin waren sich alle einig, sicher nicht zweckmässig. Die Ärzte müssen einbezogen werden, und es gilt zu vermeiden, dass die Prävention unter einem Konflikt zwischen «Medizinern» und "Sozialen» leidet.

\section{Vordringlich: gegenseitige Information}

Es geht um eine partnerschaftiiche Zusammenarbeit der verschiedenen Organisationen, auch mit solchen spezifischer Zielsetzung. Gefordert sind in erster Linie gegenseitige Information und Koordination. Die Bedeutung der Dokumentation wurde erwähnt, und dabei auch die Rolle, die das Institut für Krankenhausund Gesundheitswesen in Aarau spielen kann und gegenwärtig auch spielt.

\section{Private Dachorganisationen auf kantonaler Ebene}

Der Ruf, dass die Kantone mit der Gesetzgebung für Prävention vorwärtsmachen, wurde natürlich auch erhoben. Es wurde darauf hingewiesen, dass wir eigentlich neben der schweizerischen auch kantonale Gesundheitsligenkonferenzen haben sollten, die der föderalistischen Struktur unseres Gesundheitswesens entsprechen würden. In verschiedenen Kantonen (Waadt und Graubünden und übrigens auch Baselland) ist der Gedanke der kantonalen Gesundheitsliga schon teilweise verwirklicht.

\section{Gesundheit, letztlich ein Gut des einzelnen}

Vom Vertreter des Invalidenverbandes hervorgehoben. Auch innerhalb geschlossener Verbände können durchaus gewisse Differenzen zwischen Untergruppen wieder entstehen. Es gilt sie laufend zu überwinden.

Die Diskussion schloss mit einer optimistischen Note, abgegeben vom Vertreter der Dokumentationsstelle für Gesundheitserziehung, der darauf hinwies, dass bei geeignetem Vorgehen und bewusstem Ansprechen des einzelnen nicht nur genügend Aufgaben, sondern auch genügend Mittel für alle Partner im Gesundheitswesen vorhanden seien. 